\title{
Palynotaxonomy of Vernonanthura H. Rob. (Vernonieae, Asteraceae) species from Southeast Brazil
}

\author{
CLAUDIA B. F. MENDONÇA ${ }^{1}$, VANIA GONÇALVES-ESTEVES ${ }^{1}$, ROBERTO L. ESTEVES ${ }^{2,3}$ and \\ ALESSANDRA D. NUNES ${ }^{1}$
}

(received: May 03, 2007; accepted: July 15, 2009)

\begin{abstract}
Palynotaxonomy of Vernonanthura H. Rob. (Vernonieae, Asteraceae) species from Southeast Brazil). The present work studied the pollinic characteristics of 22 species of Vernonanthura from Southeast Brazil. The pollen grains were acetolysed, measured, described and ilustrated under light, scanning and transmission electron microscopy. Based on the results, Vernonanthura was considered an euripolinic genus as pollen grains presented distinctive characteristics, mainly related to the ornamentation, the size and apertures of the pollen grains, which may serve as a further tool in the delimitation of the species.
\end{abstract}

Key words - Asteraceae, palinology, Southeast Brazil, Vernonanthura, Vernoniinae

RESUMO - (Palinotaxonomia de espécies de Vernonanthura H. Rob. (Vernonieae, Asteraceae) ocorrentes no Sudeste do Brasil). O presente trabalho estuda os grãos de pólen de 22 espécies de Vernonanthura ocorrentes na região sudeste do Brasil. Os grãos de pólen foram acetolisados, mensurados, descritos e ilustrados sob microscopia de luz, eletrônica de varredura e de transmissão. Com base nos resultados obtidos considerou-se Vernonanthura um gênero euripolínico cujos grãos de pólen apresentaram características marcantes, principalmente relacionadas à ornamentação, ao tamanho dos grãos de pólen e às características da abertura, podendo servir como uma ferramenta a mais na delimitação das espécies.

Palavras-chave - Asteraceae, palinologia, sudeste do Brasil, Vernonanthura, Vernoniinae

\section{Introduction}

The genus Vernonanthura was described by Robinson (1992) based on species of the genus Vernonia Schreb. with which it shares some attributes, such as thyrsoid to pyramidal inflorescences, sessile heads, minute involucral bracts, flat receptacle, glabrous corolla with distinctive longitudinal resin ducts at the lobes, tailed anther bases, oblongo-ovatae, generally glandulous; cypsela with raphids from subquadrate to oblong, and, with a pollen type A, that is echinate to sublophate, tricolporate, continuous tectum, microperforated and with prominent spines (Jones 1977).

In an analysis of the generic and subtribal status of Vernonieae, Robinson (1999) stated that the pollen type A is apparently a reversion of the lophate form and is well fixed in some groups of Neotropical Vernonieae, such as Lychnophorinae, Piptocarphinae and Vernonia/ Vernonanthura.

According to Robinson (1992, 1999), Vernonanthura has 65 species, distributed through Mexico, the West Indies, Central and South America. In Brazil, there

\footnotetext{
1. Universidade Federal do Rio de Janeiro, Museu Nacional, Quinta da Boa Vista, São Cristóvão, 20940-040 Rio de Janeiro, RJ, Brazil.

2. Universidade do Estado do Rio de Janeiro, Instituto de Biologia, IBRAG/DBV, Rua São Francisco Xavier 524, Maracanã, 2550-013 Rio de Janeiro, RJ, Brazil.

3. Corresponding author: esteves.vr@gmail.com
}

are about 34 species, predominantly in Southern and Southeastern regions. Furthermore, the author emphasized that the species of typical Vernoniinae (Vernonia s.s., Vernonanthura H. Rob., Cololobus H. Rob. and Trepadonia H. Rob.) are characterized by glabrous lobes of the corola and also by a pollen grains type A.

Stutts (1988) revised the subsection Chamaedrys of the genus Vernonia s.l., setting in order the species of Vernonanthura in two subsections using, among other characters, the pollen grains, which, according to the author, belonged to the type B (echinolophate, tricolporate pollen grains, with a discontinuous tectum, microperforated, without polar lacunae). Based on Jones (1979), the author created a newly subgroup with some excluded species from Chamaedrys: the subsection Brevifoliae. Stutts' revision (1988) also closed the subsection Nudiflorae, uniting Vernonia niduflora Less. and Vernonia lucida Less. (previously subordinated to subsection Chamaedrys) on series Nudiflorae.

The present work characterized 22 species of Vernonanthura from Southeast Brazil, providing information to evaluate the recent classification proposed by Robinson (1999).

\section{Material and methods}

The pollen morphology of the 22 species of Vernonanthura that occur in Southeast Brazil was studied. 
The examined material was obtained from fertile anthers of flowers in anthesis and/or from mature buds, of exsiccated specimens belonging to the following herbaria: ESA, GUA, HB, MBM, R, RB, SPF, HUFU, VIC (acronyms according with Holmgren et al. 1990).

For each species, one specimen was chosen as standard and it is presented in the list below, indicated by an asterisk (*) placed before the collector's name. Descriptions and illustrations were also based on this standard material. When possible, up to four specimens were analyzed for comparison in order to confirm the obtained results. The permanent slides are stored at the Laboratório de Palinologia Álvaro Xavier Moreira, Departamento de Botânica do Museu Nacional/ Universidade Federal do Rio de Janeiro.

Examined specimens: Vernonanthura brasiliana (L.) H. Rob. - BRASIL. Minas Gerais: Belo Horizonte, Santa Luzia, 20-VII-1955, Luiz Roth 1455 (MBM); Diamantina, Bocaiúva, 23-VII-1998, G. Hatschbach et al. 68123 (MBM); Ituiutaba, 16-VII-1944, A. Macedo 66 (MBM); Patos, 11-IX-1963, *A. Castellos 24113 (GUA); Paraopeba, 13-VIII-1987, F.A. da Silveira 126 (VIC). V. chamaedrys (Less.) H. Rob. - BRASIL. MinAs GERAIs: Barbacena, Distrito de Pinheiro Grosso, VII-1944, *J. Vidal I-232 (R); Belo Horizonte, 8-VIII-1932, Mello Barreto 1028 (HB); Caeraneas, 2-VII-1987, H.F. Leitão Filho et al. 19415 (MBM). V. crassa (Vell.) H. Rob. - BRASIL. São PAulo: Apiaí, Barra do Chapéu; 3-VI-1994, V.C. Souza et al. 6113 (ESA); Itararé, 6-10-IX-1993, *V. C. Souza et al. 4063 (MBM); Rosana, 18-VI-1998, M.P. Manara et al. 8 (R). V. cuneifolia (Gardn.) H. Rob. - BRASIL. MinAs GERAIs: Liberdade, 15-VI-1943, M. Magalhães 2876 (HB); Uberlândia, Estação Ecológica do Panga, 27-VIII-1999, *M.T.O. Lema s.n. (HUFU21231). V. discolor (Spreng.) H. Rob. - BRASIL. EsPíRITO SANTO: Muniz Freire, 10-X-1992, G. Hatschbach et al. 57942 (MBM); Minas Gerais: Alto Caparão, 3-IX-1996, V.C. Souza et al. 12215 (ESA); Rio DE JANEIRo: Nova Friburgo, Reserva Ecológica de Macaé de Cima, 13-IX-1990, C.M.B. Correia et al. 215 (GUA); Parque Nacional da Tijuca, 3-X-2001, *C.A.L. de Oliveira 1885 (R); São PAulo: Município de Itararé, 16-VIII-1994, K.D. Barreto et al. 2891 (ESA). V. divaricata (Spreng.) H. Rob. - BRASIL. EsPíRITO SANTO: Venda Nova, 9-XI-1993, G. Hatschbach et al. 59707 (MBM); MinAs GERAIs: Diogo de Vasconcelos, 13-X-2000, A.F. Carvalho 735 (VIC); Rio DE JANEIRO: Nova Friburgo, 10-X-1993, *C.M. Vieira \& L.C. Gurken 436 (RB); São PAUlo: São Paulo, 28-IX-1951, W. Hoehne 3668 (MBM); São Paulo, Serra da Cantareira, s.d, M. Koscinski s.n. (SPF7171). V. ferruginea (Less.) H. Rob. BRASIL. Minas Gerais: Curvelo, Sucuriú, 10-VIII-1980, J.P.P. Carauta et al. 3517 (GUA); Diamantina, 19-XI-1965, *A.P. Duarte 8940 \& E. Pereira 9953 (RB); Jequitibá, 31-VII-1962, J.P. Lanna-Sobrinho 247 (GUA); Paraopeba, 2-VIII-1987, F.A. Souza 122(VIC); São Romão, 2-VIII-1982, F.C.F. Silva 161 (RB). V. ignobilis (Less.) H. Rob. - BRASIL. Minas Gerais: Gouveia, 13-XI-1971, G. Hatschbach et al. 27862 (MBM); Paracatu, 14-X-1965, *E. Pereira 10212
\& A.P. Duarte 9302 (RB). V. lindbergii (Baker) H. Rob. BRASIL. Minas Gerais: Lundréia, 6-V-1970, J. Augusto s.n. (MBM188056); SÃo PAUlO: Botucatu, 17-V-1977, *I. Gottsberger \& G. Gottsberger 21-17577 (MBM). V. lucida (Less.) H.Rob. - BRASIL. MinAs GERAIs: Conceição do Mato Dentro, 23-XI-1997, *G. Hatschbach et al. 67442 (MBM); Jaboticatubas, 16-XI-1942, M. Magi 2694 (HB); Serra do Cipó, 19-II-1968, H.S. Irwin \& H. Maxwell s.n. (HB52051). V. mariana (Mart. ex Baker) H. Rob. - BRASIL. Minas Gerais: Conceição do Mato Dentro, 30-V-1989, * $R$. Esteves \& V. Esteves 427 (R); Diamantina, 19-III-1997, G. Hatschbach et al. 66506 (MBM); Diamantina, 25-VII-1998, G. Hatschbach et al. 68252 (MBM); Presidente Kubitschek, 21-XI-1997, G. Hatschbach et al. 67376 (MBM); Serra do Cipó, 21-V-1989, R. Esteves \& V. Esteves 440 (R). V. membranacea (Gardn.) H. Rob. - BRASIL. MinAs GERAIs: Francisco Dumont, Serra do Cabral, 16-V-2001, ${ }^{*} G$. Hatschbach et al. 72203 (MBM); Joaquim Felício, Serra do Cabral, 14-V-2001, G. Hatschbach et al. 72005 (MBM); Virginópolis, 14-IX-2001, E.W. Teixeira s.n. (VIC26896). V. montevidensis (Spreng.) H. Rob. - BRASIL. MINAS GeraIs: Macieiras, 15-XI-1960, C. Angeli s.n. (GUA6280); Rio dE JAneiro: Itatiaia, 22-X-1998, R.L. Esteves 966 (R); São PAulo: Itararé, 6-10-IX-1993, *V.C. Souza \& C.M. Sakuragui 4328 (ESA); Serra da Bocaina, 20-X-1966, W. Hoehne 6145 (SPF); Votorantim, Serra de São Francisco, 25-I-1984, V.F. Ferreira 3272 (GUA). V. mucronulata (Less.) H. Rob. - BRASIL. MinAs Gerais: Serra do Curral, VII-1949, *J. Vidal s.n. (R113249); São PAUlo: Botucatu, 11-VII-1974, L. Gottsburger s.n. (MBM267217); Itararé, 10-IX-1993, V.C. Souza \& C.M. Sakuragui 4127 (ESA); São Paulo, 07-VIII-1946, W. Hoehne s.n. (SPF14198); Votorantim, Serra de São Francisco, 25-I-1984, V.F. Ferreira 3243 (GUA). V. oligolepis (Sch. Bip. ex Baker) H. Rob. - BRASIL. São PAulo: Assis, 20-25-II-1988, H.F. Leitão-Filho et al. 20658 (MBM); Itapeva, I-1958, G. Vidal s.n. (R113580); Itararé, 7-II-1991, *C.A. de M. Scaramuzza \& V.C. Souza 2701 (MBM). V. paludosa (Gardn.) H. Rob. - BRASIL. RIO DE JANEIRO: Itatiaia, 7-VIII-1926, A. J. Sampaio 4816 (R); ibidem, 3-VI-1902, *P. Dusén 454 (R). V. petiolaris (DC.) H. Rob. - BRASIL. MinAS GeraIs: Barbacena, VII-1944, J. Vidal 269 (R); Liberdade, 15-VI-1943, M. Magalhães 4394 (RB); RiO DE JANEIRO: Itatiaia, Campo Belo, 22-VII-1906, E. Hermmendoff 503 (R); Nova Friburgo, Reserva Ecológica Macaé de Cima, 13-IX-1988, C.M.B. Correia 11 et al. (GUA); Teresópolis, Parque Nacional da Serra dos Órgãos, VIII-1952, *J. Vidal 182 (R). V. phosphorica (Vell.) H. Rob. - BRASIL. EsPÍRITO SANTO: Alfredo Chaves, 7-VII-1996, *G. Hatschbach et al. 65250 (MBM); Rio DE JANEIRO: Bom Jardim, 24-VIII-1984, J.P.P. Carauta et al. (GUA); Paraíba do Sul, 24-VII-1984, J.P.P. Carauta 4781 et al. (GUA); Teresópolis, Cascata dos Amores, 7-VII-1984, E.F. Paciornil 55 (MBM); São PAUlo: São Paulo, 25-VII-1944, W. Hoehne 1417 (SPF). V. puberula (Less.) H. Rob. - BRASIL. RIO DE JANEIRO: Nova Friburgo, 19-XII-1887, *Glaziou 17022 (R); SÃo PAULO: 
Cunha, 10-VII-1980, A. Custodio-Filho 285 (MBM). V. rigiophylla (Kuntze) H. Rob. - BRASIL. MinAs GERAIs: São Roque de Minas, 18-VII-1995; *J.N. Nakajima et al. 1214 (R); Serra de São José, VI-1896, A. Silveira 1257 (RB). V. viscidula (Less.) H. Rob. - BRASIL. MinAs GERAIs: Belo Horizonte, 19-V-1933, M. Barreto 4233 (R); São Roque de Minas, 14-V-1933, R. Romero et al. 2292 (R); Serra do Palmital, 20-VI-1884, *Glaziou 15060 (R). V. westiniana (Less.) H. Rob. - BRASIL. Minas Gerais: Caldas, VII-1914, A.F. Regnell 1245 (R); São Roque de Minas, 12-I-1996, R. Romero et al. 3289 (MBM/R); RiO DE JANEIRO: Japeri, 21-VII-2001, *M. Frigoleto s.n. (GUA47702); São PAulo: Itararé, V.C. Souza et al. 2173 (MBM); São Paulo, 10-IV-1955, W. Hoehne 4081 (MBM).

For the study under light microscope (LM), the pollen grains were submitted to the Erdtman (1952) acetolytic method, measured up to seven days later (Salgado-Labouriau 1973), and photomicrographed. For scanning electron microscopy (SEM), non acetolysed pollen samples were mounted on stubs and coated with gold-platinum. The analysis was done in a JSM-5310 microscope in the Laboratório de Ultraestrutura Celular Hertha Meyer, Instituto de Biofísica, Universidade Federal do Rio de Janeiro. For transmission electron microscopy (TEM), the anthers were dehydrated by $2 \%$ glutaraldehyde solution, embedded in Spurr resin, and sectioned with a diamond knife on Ultra-microtome U2088.

A minimum of 25 pollen grains per sample were measured in equatorial view (polar diameter $=\mathrm{PD}$ and equatorial diameter $=\mathrm{ED}$ ) in the light microscope (LM). In all the measurements, the spines were included. Statistical procedures were carried out, calculating the arithmetic mean $(\bar{x})$; the standard deviation of the mean $\left(s_{x}\right)$ and the confidence interval at $95 \%$. For the measurements of the other characteristics, such as equatorial diameter in polar view (EDPV), side of apocolpium (SA), endoapertures size and layers of exine, the arithmetic mean of 10 measurements were calculated. The same procedure was applied for the measurements of the pollen grain diameters of the comparison material.

The terminology used for pollen descriptions followed the criteria of Punt et al. (2007); the denomination of the polar area and the dimensions of the apertures are in accordance with the classification for the polar area index established by Faegri \& Iversen (1966).

\section{Results}

The pollen grains (figures 1-99) were medium $(25.0 \mu \mathrm{m}$ to $50.0 \mu \mathrm{m})$ to large sized $(50.0 \mu \mathrm{m}$ to $100.0 \mu \mathrm{m}$ ) only in V. crassa, V. ignobilis, V. lucida, $V$. oligolepis and $V$. viscidula, isopolar, oblate spheroidal (table 1), subtriangular to subcircular amb, very small polar area (V. crassa, V. ferruginea, V. mucronulata, $V$. oligolepis, $V$. paludosa, V. petiolaris and V. puberula) or small (table 2), 3-colporate with a subechinolophate surface (figures 2, 8, 10, 13, 19, 22, 30, 42, 54, 57, 70, 88, 94).

Aperture -3 colpori, delimited by sinuous muri. Colpus long to very long (table 3 ), the biggest 24.3 to $27.7 \mu \mathrm{m}$

Table 1. Pollen measurements $(\mu \mathrm{m})$ in equatorial view of Vernonanthura species. $\left(\bar{x}=\right.$ arithmetic mean; $s_{x}=$ standard deviation of medium; $\mathrm{CI}$ = confidence interval; $\mathrm{P}=$ Polar diameter; $\mathrm{E}=$ equatorial diameter).

\begin{tabular}{|c|c|c|c|c|c|}
\hline \multirow{2}{*}{ Species } & \multicolumn{2}{|c|}{ Polar diameter } & \multicolumn{2}{|c|}{ Equatorial diameter } & \multirow[b]{2}{*}{$\mathrm{P} / \mathrm{E}$} \\
\hline & $\begin{array}{l}\text { Range variation } \\
\qquad\left(\bar{x} \pm s_{x}\right)\end{array}$ & CI 95\% & $\begin{array}{l}\text { Range variation } \\
\qquad\left(\bar{x} \pm s_{x}\right)\end{array}$ & CI 95\% & \\
\hline V. brasiliana & $42.5(43.9 \pm 0.2) 46.2$ & $43.5-44.3$ & $45.0(45.8 \pm 0.2) 48.7$ & $45.4-46.2$ & 0.95 \\
\hline V. chamaedrys & $46.3(48.8 \pm 0.3) 51.3$ & $48.2-49.4$ & $52.5(55.2 \pm 0.3) 58.8$ & $54.6-55.8$ & 0.88 \\
\hline V. crassa & $51.2(52.6 \pm 0.1) 53.7$ & $52.4-52.8$ & $57.5(59.4 \pm 0.2) 60.0$ & $59.0-59.8$ & 0.88 \\
\hline V. cuneifolia & $45.0(45.6 \pm 0.2) 47.5$ & $45.2-46.0$ & $50.0(51.2 \pm 0.2) 53.7$ & $50.8-51.6$ & 0.89 \\
\hline V. discolor & $36.2(37.8 \pm 0.2) 40.0$ & $37.4-38.2$ & $40.0(41.9 \pm 0.2) 43.7$ & $41.5-42.3$ & 0.90 \\
\hline V. divaricata & $40.0(41.1 \pm 0.2) 42.5$ & $40.7-41.5$ & $40.0(42.8 \pm 0.3) 45.0$ & $42.2-43.4$ & 0.96 \\
\hline V. ferruginea & $40.0(41.8 \pm 0.2) 42.5$ & $41.4-42.2$ & $45.0(45.7 \pm 0.2) 47.5$ & $45.3-46.1$ & 0.91 \\
\hline V. ignobilis & $51.2(53.2 \pm 0.2) 55.0$ & $52.8-53.6$ & $53.7(56.1 \pm 0.2) 57.5$ & $55.7-56.5$ & 0.94 \\
\hline V. lindbergii & $43.7(45.0 \pm 0.2) 46.2$ & $44.6-45.4$ & $48.7(50.2 \pm 0.2) 52.5$ & $49.8-50.6$ & 0.89 \\
\hline V. lucida & $52.5(54.5 \pm 0.2) 57.5$ & $54,1-54,9$ & $57.5(58.5 \pm 0.2) 60.0$ & $58.1-58.9$ & 0.93 \\
\hline V. mariana & $37.5(39.3 \pm 0.2) 40.0$ & 38.9-39.7 & $40.0(41.9 \pm 0.2) 43.8$ & $41.5-42.3$ & 0.93 \\
\hline
\end{tabular}


continuation

\begin{tabular}{|c|c|c|c|c|c|}
\hline \multirow{2}{*}{ Species } & \multicolumn{2}{|c|}{ Polar diameter } & \multicolumn{2}{|c|}{ Equatorial diameter } & \multirow[b]{2}{*}{$\mathrm{P} / \mathrm{E}$} \\
\hline & $\begin{array}{l}\text { Range variation } \\
\qquad\left(\bar{x} \pm s_{x}\right)\end{array}$ & CI 95\% & $\begin{array}{l}\text { Range variation } \\
\qquad\left(\bar{x} \pm s_{x}\right)\end{array}$ & CI 95\% & \\
\hline V. membranacea & $40.0(41.4 \pm 0.2) 43.8$ & $41.0-41.8$ & $42.5(43.8 \pm 0.2) 45.0$ & $43.4-44.2$ & 0.94 \\
\hline V. montevidensis & $43.7(45.7 \pm 0.2) 47.5$ & $45.3-46.1$ & $46.2(48.8 \pm 0.2) 50.0$ & $48.4-49,2$ & 0.93 \\
\hline V. mucronulata & $47.5(48.3 \pm 0.2) 50.0$ & $47.9-48.7$ & $50.0(52.7 \pm 0.2) 55.0$ & $52,3-53.1$ & 0.91 \\
\hline V. oligolepis & $47.5(52.1 \pm 0.3) 53.8$ & $51.6-52.7$ & $52.5(54.5 \pm 0.2) 56.3$ & $54.1-54.9$ & 0.95 \\
\hline V. paludosa & $42.5(44.2 \pm 0.2) 45.0$ & $43.8-44.6$ & $47.5(48.2 \pm 0.2) 51.2$ & 47.8-48.6 & 0.91 \\
\hline V. petiolaris & $43.7(46.7 \pm 0.2) 47.5$ & $46.3-47.1$ & $47.5(50.1 \pm 0.3) 52.5$ & $49.5-50.7$ & 0.93 \\
\hline V. phosphorica & $45.0(46.5 \pm 0.2) 47.5$ & $46.1-46.9$ & $50.0(51.0 \pm 0.2) 52.5$ & 50.6-51.4 & 0.91 \\
\hline V. puberula & $47.5(49.4 \pm 0.3) 51.3$ & $48.8-50.0$ & $51.3(53.7 \pm 0.2) 55.0$ & $53.3-54.1$ & 0.91 \\
\hline V. rigiophylla & $46.3(48.1 \pm 0.3) 51.3$ & $47.5-48.7$ & $47.5(51.1 \pm 0.3) 53.8$ & $50.5-51.7$ & 0.94 \\
\hline V. viscidula & $47.5(50.5 \pm 0.2) 52.5$ & $50.1-50.9$ & $52.5(55.9 \pm 0.3) 58.7$ & $55.3-56.5$ & 0.90 \\
\hline V. westiniana & $40.0(42.3 \pm 0.2) 43.7$ & $41.9-42.7$ & $45.0(45.9 \pm 0.2) 48.7$ & $45.5-46.3$ & 0.92 \\
\hline
\end{tabular}

Table 2. Pollen measurements $(\mu \mathrm{m})$ in polar view of Vernonanthura species. $\left(\bar{x}=\right.$ arithmetic mean; $s_{x}=$ medium standard; $\mathrm{CI}$ = confidence interval; SA = apocolpium side; $\mathrm{EDPV}=$ equatorial diameter in polar view and polar area index (PAI)).

\begin{tabular}{|c|c|c|c|c|}
\hline \multirow[b]{2}{*}{ Species } & Equatorial diameter & SA & \multirow[b]{2}{*}{ PAI } & \multirow{2}{*}{$\begin{array}{l}\text { Polar area } \\
\text { SA/EDPV }\end{array}$} \\
\hline & $\begin{array}{l}\text { Range variation } \\
\qquad\left(\bar{x} \pm s_{x}\right)\end{array}$ & Range variation $(\bar{x})$ & & \\
\hline V. brasiliana & 46.2 (48.6) 50.0 & 15.0 (16.5) 17.5 & small & 0.33 \\
\hline V. chamaedrys & 45.0 (52.5) 53.8 & $12.5(15.0) 15.0$ & small & 0.28 \\
\hline V. crassa & $57.5(60.8) 62.5$ & $10.0(12.5) 15.0$ & very small & 0.20 \\
\hline V. cuneifolia & 50.0 (50.6) 52.5 & $21.2(22.0) 22.5$ & small & 0.43 \\
\hline V. discolor & $40.0(40.9) 42.5$ & $10.0(11.0$ (12.5 & small & 0.26 \\
\hline V. divaricata & $40.0(42.5) 43.7$ & $12.5(14.2) 15.0$ & small & 0.33 \\
\hline V. ferruginea & $42.5(44.2) 45.0$ & $7.5(9.9) 11.2$ & very small & 0.22 \\
\hline V. ignobilis & 57.5 (58.7) 60.0 & 15.0 (17.9) 20.0 & small & 0.30 \\
\hline V. lindbergii & 47.5 (49.5) 50.0 & 12.5 (14.1) 15.0 & small & 0.28 \\
\hline V. lucida & $55.0(56.8) 57.5$ & 15.5 (16.7) 17.5 & small & 0.29 \\
\hline V. mariana & 42.5 (43.4) 45.0 & 10.0 (11.1) 12.5 & small & 0.25 \\
\hline V. membranacea & 41.3 (43.4) 45.0 & 10.0 (11.4) 12.5 & small & 0.26 \\
\hline V. montevidensis & $46.2(48.5) 51.2$ & $11.2(13.4) 15.0$ & small & 0.27 \\
\hline V. mucronulata & $51.2(52.6) 53.7$ & 12.5 (12.9) 13.7 & very small & 0.24 \\
\hline V. oligolepis & 52.5 (53.9) 55.0 & $10.0(12.3) 13.8$ & very small & 0.22 \\
\hline V. paludosa & 43.7 (46.4) 47.5 & $10.0(11.5) 12.5$ & very small & 0.24 \\
\hline V. petiolaris & $46.2(48.6) 50.0$ & $10.0(11.7) 12.5$ & very small & 0.24 \\
\hline V. phosphorica & 48.7 (50.6) 52.5 & 12.5 (13.7) 15.0 & small & 0.27 \\
\hline V. puberula & 52.5 (54.4) 57.5 & $11.3(12.8) 15.0$ & very small & 0.23 \\
\hline V. rigiophylla & 48.8 (52.9) 56.3 & 12.5 (13.6) 15.0 & small & 0.25 \\
\hline V. viscidula & 48.7 (53.4) 55.0 & $20.0(24.0) 25.0$ & small & 0.44 \\
\hline V. westiniana & 43.7 (45.5) 47.5 & $15.0(16.5) 17.5$ & small & 0.36 \\
\hline
\end{tabular}


(V. mucronulata and $V$. oligolepis); the smallest ones 20.8 to $23.5 \mu \mathrm{m}$ long ( $V$. discolor, $V$. mariana and $V$. montevidensis); colpus wide varying between $3.8 \mu \mathrm{m}$ (V. brasiliana) and $7.4 \mu \mathrm{m}$ (V. lucida and V. puberula). The endoaperture is lalongate or slightly lolongate in $V$. ignobilis (table 3). Furthermore, in some species the endoapertures present a median constriction, like in $V$. crassa, $V$. cuneifolia, $V$. divaricata, $V$. montevidensis, $V$. mucronulata, $V$. oligolepis, $V$. rigiophylla and $V$. viscidula (figures 11, 15, 23, 55, 59, 63, 84, 91). In V. brasiliana, the muri that delimit the colpus are high difficulting the colpus observation (figures 3, 4). In SEM, ornamented membrane was observed covering the colpus (figures $14,32,44,56,60,62)$.

Exine - Subequinolophate, with a simplicolumellate. The sexine can be composed of very sinuous walls ( $V$. brasiliana, V. chamaedrys, V. lucida, V. montevidensis, $V$. mucronulata, $V$. rigiophylla, and $V$. westiniana) or slightly sinuous in the other species. The muri may tend towards the lophate pattern, although without closed lacunae delimiting them, which can be better observed in SEM (figures 10, 16, 22, 27, 42, 52, 56, 66, 72, 77, 82, 85 and 89). In the latter pattern were found regions between the muri that may be broad or narrow, psilate to lightly scabrate. In the apocolpus region, the muri may be continuous (in the majority of the species) or discontinuous (figures 14, 18, 32, 36, 47, 62, 74, 82, 89 ) and their concentration also varies, being possible to find muri only delimiting the apertures ( $V$. ignobilis, $V$. oligolepis). The muri thickness in the apocolpus area varied from 1.5 in $V$. membranacea to $4.0 \mu \mathrm{m}$ in $V$. crassa. In the mesocolpus, the organization of the muri varies and their discontinuity in some species is so great that isolated spines may be found, such as in $V$. ignobilis and $V$. lindbergii (figures 34, 39). The spines show variations in length and width (table 3). The longest spines (7.3 to $8.0 \mu \mathrm{m}$ ) were found in $V$. lindbergii, V. mariana, V. phosphorica and V. puberula and the shortest ones $(\mathrm{ca} .3 .8 \mu \mathrm{m})$ in $V$. brasiliana, $V$.

Table 3. Average $(\mu \mathrm{m})$ size of the apertures and morphometric data of the exine of Vernonanthura species $(n=10)$.

\begin{tabular}{|c|c|c|c|c|c|c|c|c|c|c|c|}
\hline \multirow{2}{*}{ Species } & \multicolumn{2}{|c|}{ Colpus } & \multicolumn{2}{|c|}{ Endoaperture } & \multicolumn{3}{|c|}{ Exine layers } & \multirow{2}{*}{$\begin{array}{l}\text { Muri } \\
\text { width }\end{array}$} & \multicolumn{3}{|c|}{ Spine } \\
\hline & length & width & length & width & exine & sexine & nexine & & length & width & dist. \\
\hline V. brasiliana & 23.5 & 3.8 & 4.5 & 8.5 & 10.0 & 9.0 & 1.0 & 3.4 & 5.0 & 2.0 & 7.5 \\
\hline V. chamaedrys & 23.0 & 4.5 & 5.0 & 10.0 & 10.7 & 9.4 & 1.3 & 2.8 & 5.8 & 2.0 & 8.7 \\
\hline V. crassa & 26.8 & 6.5 & 7.5 & 10.3 & 12.3 & 11.1 & 1.2 & 4.0 & 6.7 & 1.9 & 9.2 \\
\hline V. cuneifolia & 22.1 & 4.2 & 7.0 & 10.3 & 9.9 & 8.9 & 1.0 & 3.6 & 5.0 & 2.0 & 7.8 \\
\hline V. discolor & 20.8 & 4.7 & 7.1 & 8.3 & 8.2 & 7.1 & 1.0 & 2.0 & 3.8 & 2.0 & 6.2 \\
\hline V. divaricata & 24.5 & 4.5 & 4.7 & 12.0 & 10.5 & 9.5 & 1.0 & 3.5 & 5.4 & 1.4 & 8.7 \\
\hline V. ferruginea & 26.4 & 5.4 & 5.0 & 14.4 & 10.2 & 9.2 & 1.0 & 2.7 & 5.4 & 1.8 & 8.4 \\
\hline V. ignobilis & $24 ., 0$ & 6.0 & 11.8 & 11.2 & 12.8 & 11.0 & 1.8 & 2.4 & 6.5 & 2.2 & 10.4 \\
\hline V. lindbergii & 24.7 & 6.0 & 7.0 & 10.4 & 12.4 & 11.4 & 1.0 & 2.0 & 7.4 & 1.5 & 8.7 \\
\hline V. lucida & 25.4 & 7.4 & 7.4 & 13.0 & 11.0 & 9.8 & 1.2 & 2.0 & 5.3 & 2.0 & 9.0 \\
\hline V. mariana & 21.5 & 4.9 & 5.4 & 10.3 & 12.5 & 11.5 & 1.0 & 2.0 & 7.5 & 2.0 & 9.0 \\
\hline V. membranacea & 23.5 & 5.0 & 4.5 & 10.5 & 12.0 & 11.0 & 1.0 & 1.5 & 5.0 & 2.0 & 8.0 \\
\hline V. montevidensis & 22.3 & 5.0 & 3.9 & 8.5 & 9.7 & 8.6 & 1.0 & 1.7 & 5.7 & 1.9 & 7.2 \\
\hline V. mucronulata & 27.6 & 6.2 & 6.6 & 10.3 & 12.4 & 11.4 & 1.1 & 2.2 & 6.2 & 2.1 & 10.6 \\
\hline V. oligolepis & 27.7 & 5.5 & 7.4 & 15.2 & 11.8 & 10.5 & 1.3 & 3.3 & 5.8 & 2.0 & 7.7 \\
\hline V. paludosa & 26.5 & 5.5 & 6.7 & 9.0 & 12.6 & 11.4 & 1.2 & 2.4 & 6.4 & 2.0 & 9.2 \\
\hline V. petiolaris & 26.7 & 6.5 & 5.3 & 8.7 & 11.1 & 10.1 & 1.1 & 2.2 & 6.2 & 2.0 & 10.0 \\
\hline V. phosphorica & 24.3 & 5.2 & 6.0 & 11.6 & 12.1 & 10.9 & 1.1 & 2.0 & 7.3 & 1.9 & 9.2 \\
\hline V. puberula & 26.7 & 7.4 & 8.0 & 12.0 & 13.0 & 11.7 & 1.3 & 3.4 & 8.0 & 2.3 & 9.7 \\
\hline V. rigiophylla & 23.0 & 5.7 & 5.0 & 10.8 & 11.5 & 10.5 & 1.0 & 3.5 & 6.0 & 2.5 & 10.2 \\
\hline V. viscidula & 23.3 & 5.3 & 6.1 & 10.6 & 11.8 & 10.6 & 1.1 & 2.3 & 5.5 & 2.0 & 9.8 \\
\hline V. westiniana & 23.5 & 4.6 & 5.7 & 10.5 & 11.3 & 10.3 & 1.1 & 2.0 & 6.1 & 2.9 & 8.9 \\
\hline
\end{tabular}



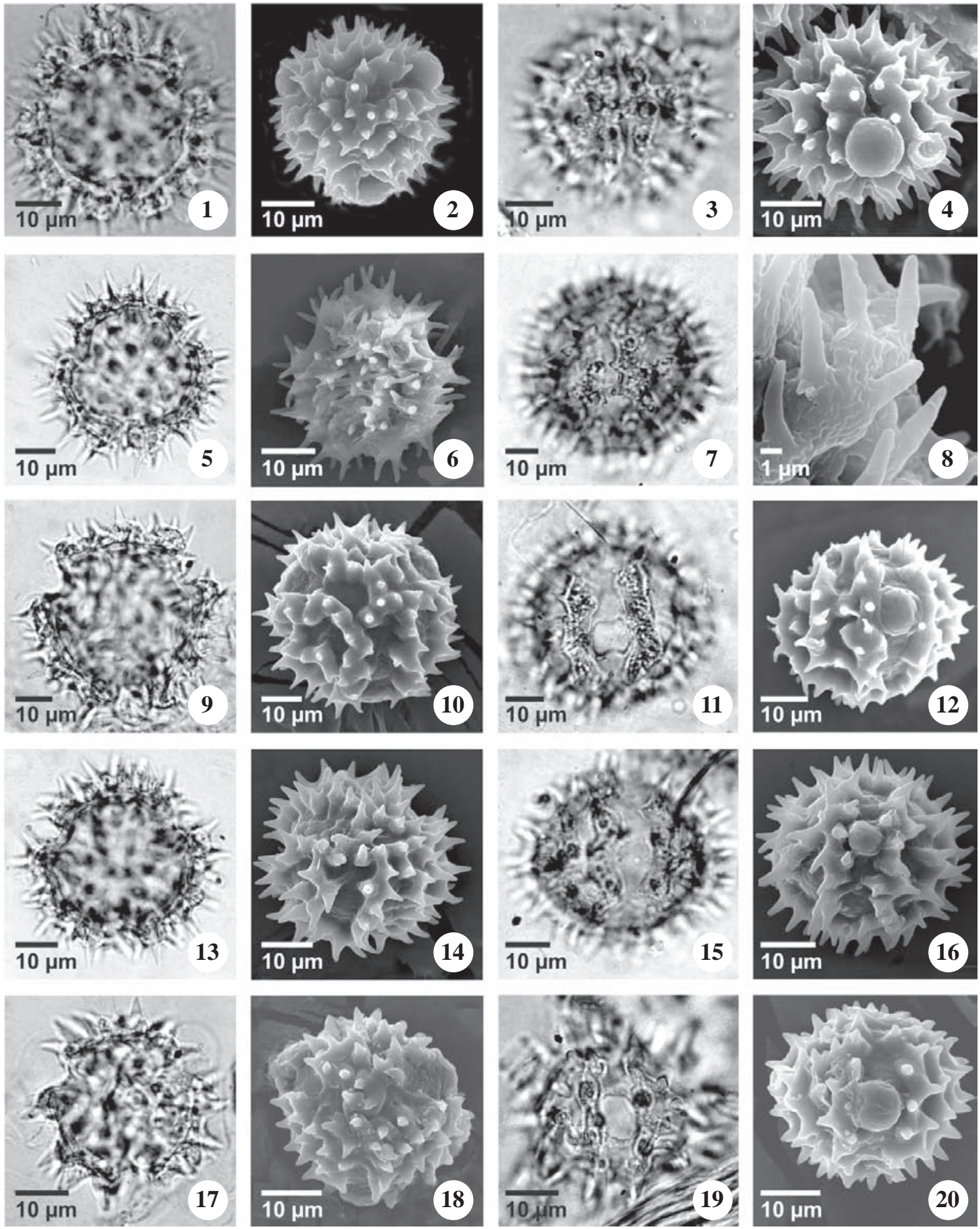

Figures 1-20. LM and SEM photomicrographs of the pollen grains of Vernonanthura. 1-4. V. brasiliana (A. Castellos 24113) - Polar view. 1. Cross section. 2. Surface of apocolpium. Equatorial view. 3-4. Aperture. 5-8. V. chamaedrys (J. Vidal I-232) - Polar view. 5. Cross section. 6. Surface of apocolpium. Equatorial view. 7. Aperture. 8. Detail of surface. 9-12. V. crassa (V.C. Souza et al. 4063) - Polar view. 9. Cross section. 10. Surface of apocolpium. 11-12. Equatorial view. Aperture. 13-16. V. cuneifolia (M.T.O. Lema s.n. HUFU21231) - Polar view. 13. Cross section. 14. Surface of apocolpium. 15-16. Aperture. 17-20. V. discolor (C.A.L. de Oliveira 1885) - Polar view. 17. Cross section. 18. Surface of apocolpium. 19-20. Equatorial view. Aperture. 

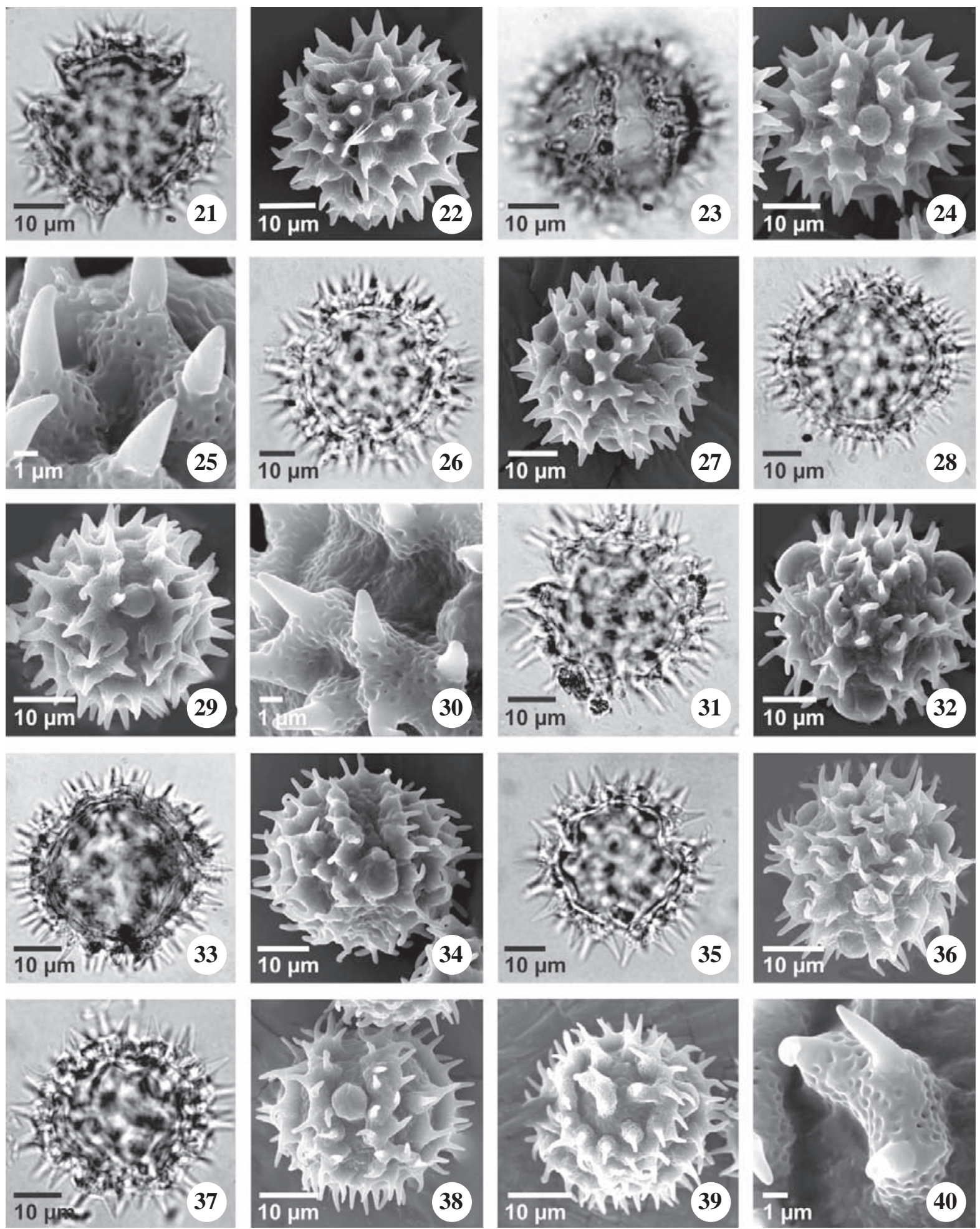

Figures 21-40. LM and SEM photomicrographs of the pollen grains of Vernonanthura. 21-25. V. divaricata (C.M. Vieira 436 \& L.C. Gurken) - Polar view. 21. Cross section. 22. Surface of apocolpium. Equatorial view. 23-24. Aperture. 25. Surface. 26-30. V. ferruginea (A.P. Duarte 8940 \& E. Pereira 9953) - Polar view. 26. Cross section. 27. Surface of apocolpium. Equatorial view. 28. Cross section. 29. Aperture. 30. Surface. 31-34. V. ignobilis (E. Pereira 10212 \& A.P. Duarte 9302) - Polar view. 31. Cross section. 32. Surface of apocolpium. Equatorial view. 33. Cross section. 34. Aperture. 35-40. V. lindbergii (I. Gottsberger \& G. Gottsberger 21-17577) - Polar view. 35. Cross section. 36. Surface of apocolpium. Equatorial view. 37. Cross section. 38. Aperture. 39. Mesocolpium. 40. Detail of surface. 
cuneifolia, V. discolor and V. membranacea. The width of the spines ranged between 1.4 ( $V$. divaricata) and $2.9 \mu \mathrm{m}$ ( $V$. westiniana). The distance between the spines varies widely, ranging from 6.2 (V. discolor) to $10.6 \mu \mathrm{m}$ (V. mucronulata). The nexine is always thinner than the sexine (table 3). In SEM, the differences in quantity and size of the perforations can be better observed at the base of the spines, and also the details of the muri previously described, as well as the presence of perforations throughout their extension, with spines which are apart in their superior part. In TEM (figure 99), long, thick, solid columella can be observed supporting spines, which are linked by a thick tectum.

Comments - Table 4 shows that the results found in the specimens for comparison mostly present values of polar

Table 4. Measures $(\mu \mathrm{m})$ of pollen grains of comparison material $(n=10)$. $(\mathrm{P}=$ polar diameter; $\mathrm{E}=$ equatorial diameter; $\mathrm{EDPV}=$ equatorial diameter in polar view; $\mathrm{SA}=$ apocolpium side and polar area index (PAI)).

\begin{tabular}{|c|c|c|c|c|c|c|c|}
\hline Especimens & $\begin{array}{c}\text { Polar } \\
\text { diameter } \\
\bar{x}\end{array}$ & $\begin{array}{c}\text { Equatorial } \\
\text { diameter } \\
\bar{x}\end{array}$ & $\mathrm{P} / \mathrm{E}$ & Shape & $\begin{array}{c}\text { EDPV } \\
\bar{x}\end{array}$ & $\begin{array}{c}\text { SA } \\
\bar{x}\end{array}$ & PAI \\
\hline \multicolumn{8}{|l|}{ V. brasiliana } \\
\hline Luiz Roth 1455 & 42.8 & 46.1 & 0.92 & oblate spheroidal & 44.6 & 15.0 & 0.33 \\
\hline G. Hatschbach et al. 68123 & 43.0 & 45.7 & 0.94 & oblate spheroidal & 44.7 & 11.2 & 0.25 \\
\hline A. Macedo 66 & 43.5 & 46.9 & 0.92 & oblate spheroidal & 48.0 & 13.7 & 0.28 \\
\hline F.A. da Silveira 126 & 40.5 & 43.0 & 0.94 & oblate spheroidal & 42.8 & 13.0 & 0.30 \\
\hline \multicolumn{8}{|l|}{ V. chamaedrys } \\
\hline Mello Barreto 1028 & 46.6 & 58.2 & 0.80 & suboblate & 48.3 & 16.7 & 0.34 \\
\hline H.F. Leitão Filho et al. 19415 & 47.5 & 55.9 & 0.84 & suboblate & 48.4 & 14.1 & 0.29 \\
\hline \multicolumn{8}{|l|}{ V. crassa } \\
\hline V.C. Souza et al. 6113 & 54.0 & 59.0 & 0.91 & oblate spheroidal & 61.2 & 14.0 & 0.22 \\
\hline M.P. Manara et al. 8 & 53.2 & 58.3 & 0.91 & oblate spheroidal & 60.9 & 15.6 & 0.25 \\
\hline \multicolumn{8}{|l|}{ V. cuneifolia } \\
\hline M. Magalhães 2876 & 47.0 & 51.7 & 0.90 & oblate spheroidal & 48.1 & 20.0 & 0.41 \\
\hline \multicolumn{8}{|l|}{ V. discolor } \\
\hline G. Hatschbach et al. 57942 & 38.3 & 41.7 & 0.91 & oblate spheroidal & 43.3 & 10.8 & 0.24 \\
\hline V.C. Souza et al. 12215 & 40.1 & 42.6 & 0.94 & oblate spheroidal & 41.6 & 10.1 & 0.24 \\
\hline C.M.B. Correia et al. 215 & 38.5 & 41.9 & 0.91 & oblate spheroidal & 40.9 & 11.4 & 0.27 \\
\hline K.D. Barreto et al. 2891 & 39.2 & 42.1 & 0.93 & oblate spheroidal & 43.0 & 11.1 & 0.25 \\
\hline \multicolumn{8}{|l|}{ V. divaricata } \\
\hline G. Hatschbach et al. 59707 & 41.6 & 49.8 & 0.83 & suboblate & 43.1 & 13.5 & 0.31 \\
\hline A.F. Carvalho 735 & 40.1 & 44.6 & 0.90 & oblate spheroidal & 44.2 & 13.9 & 0.31 \\
\hline W. Hoehne 3668 & 40.9 & 44.5 & 0.91 & oblate spheroidal & 41.9 & 12.9 & 0.30 \\
\hline M. Koscinski s.n. (SPF7171) & 41.3 & 43.8 & 0.94 & oblate spheroidal & 42.5 & 13.9 & 0.32 \\
\hline \multicolumn{8}{|l|}{ V. ferruginea } \\
\hline J.P.P. Carauta et al. 3517 & 41.0 & 46.2 & 0.88 & oblate spheroidal & 44.7 & 11.7 & 0.26 \\
\hline J.P. Lanna-Sobrinho 247 & 41.6 & 45.6 & 0.91 & oblate spheroidal & 42.6 & 10.7 & 0.25 \\
\hline F.A. Souza 122 & 42.4 & 46.2 & 0.91 & oblate spheroidal & 43.9 & 12.0 & 0.27 \\
\hline F.C.F. Silva 161 & 40.3 & 45.0 & 0.89 & oblate spheroidal & 43.0 & 13.9 & 0.32 \\
\hline \multicolumn{8}{|l|}{ V. ignobilis } \\
\hline G. Hatschbach et al. 27862 & 53.0 & 56.1 & 1.05 & prolate spheroidal & 58.7 & 17.9 & 0.30 \\
\hline \multicolumn{8}{|l|}{$V$. lindbergii } \\
\hline J. Augusto s.n. (MBM188056) & 45.2 & 51.9 & 0.87 & suboblate & 49.6 & 15.0 & 0.30 \\
\hline \multicolumn{8}{|l|}{ V. lucida } \\
\hline M. Magi 2694 & 55.9 & 58.1 & 0.96 & oblate spheroidal & 56.3 & 16.5 & 0.29 \\
\hline
\end{tabular}


continuation

\begin{tabular}{|c|c|c|c|c|c|c|c|}
\hline Especimens & $\begin{array}{c}\text { Polar } \\
\text { diameter } \\
\overline{\bar{x}}\end{array}$ & $\begin{array}{c}\text { Equatorial } \\
\text { diameter } \\
\overline{\bar{X}}\end{array}$ & $\mathrm{P} / \mathrm{E}$ & Shape & $\begin{array}{c}\text { EDPV } \\
\bar{x}\end{array}$ & $\begin{array}{l}\mathrm{SA} \\
\bar{x}\end{array}$ & PAI \\
\hline H.S. Irwin e H. Maxwell s.n. (HB52051) & 56.9 & 58.2 & 0.97 & oblate spheroidal & 57.0 & 16.9 & 0.29 \\
\hline \multicolumn{8}{|l|}{ V. mariana } \\
\hline G. Hatschbach et al. 66506 & 38.6 & 40.5 & 0.95 & oblate spheroidal & 42.6 & 11.2 & 0.26 \\
\hline G. Hatschbach et al. 68252 & 39.9 & 42.6 & 0.93 & oblate spheroidal & 43.9 & 12.8 & 0.29 \\
\hline G. Hatschbach et al. 67376 & 39.4 & 41.2 & 0.95 & oblate spheroidal & 44.0 & 12.0 & 0.27 \\
\hline R. Esteves e V. Esteves 440 & 40.2 & 43.0 & 0.93 & oblate spheroidal & 42.2 & 12.5 & 0.29 \\
\hline \multicolumn{8}{|l|}{ V. membranacea } \\
\hline G. Hatschbach et al. 72005 & 40.0 & 44.8 & 0.89 & oblate spheroidal & 44.0 & 10.6 & 0.24 \\
\hline E.W. Teixeira s.n. (VIC26896) & 40.7 & 44.1 & 0.92 & oblate spheroidal & 43.4 & 12.2 & 0.28 \\
\hline \multicolumn{8}{|l|}{ V. montevidensis } \\
\hline C. Angeli s.n. (GUA6280) & 46.6 & 50.5 & 0.92 & oblate spheroidal & 49.5 & 14.0 & 0.28 \\
\hline R.L. Esteves 966 & 47.4 & 49.4 & 0.95 & oblate spheroidal & 51.6 & 14.2 & 0.27 \\
\hline W. Hoehne 6145 & 45.9 & 48.2 & 0.95 & oblate spheroidal & 51.4 & 14.3 & 0.27 \\
\hline V.F. Ferreira 3272 & 47.5 & 49.1 & 0.96 & oblate spheroidal & 51.5 & 14.5 & 0.28 \\
\hline \multicolumn{8}{|l|}{ V. mucronulata } \\
\hline L. Gottsburger s.n. (MBM267217) & 50.3 & 53.2 & 0.94 & oblate spheroidal & 52.8 & 14.1 & 0.26 \\
\hline V.C. Souza e C.M. Sakuragui 4127 & 49.1 & 50.9 & 0.96 & oblate spheroidal & 50.1 & 14.0 & 0.27 \\
\hline W. Hoehne s.n. (SPF14198) & 49.8 & 53.3 & 0.93 & oblate spheroidal & 50.9 & 11.3 & 0.22 \\
\hline V.F. Ferreira 3243 & 48.9 & 54.3 & 0.90 & oblate spheroidal & 55.0 & 14.5 & 0.26 \\
\hline \multicolumn{8}{|l|}{ V. oligolepis } \\
\hline H.F. Leitão-Filho et al. 20658 & 53.1 & 55.9 & 0.94 & oblate spheroidal & 55.4 & 18.3 & 0.33 \\
\hline G. Vidal s.n. (R113580) & 48.8 & 52.9 & 0.92 & oblate spheroidal & 53.4 & 13.8 & 0.25 \\
\hline \multicolumn{8}{|l|}{ V. paludosa } \\
\hline A.J. Sampaio 4816 & 43.0 & 47.8 & 0.89 & oblate spheroidal & 46.9 & 11.3 & 0.24 \\
\hline \multicolumn{8}{|l|}{ V. petiolaris } \\
\hline J. Vidal 269 & 47.1 & 50.2 & 0.93 & oblate spheroidal & 50.5 & 11.0 & 0.21 \\
\hline M. Magalhães 4394 & 45.3 & 49.3 & 0.91 & oblate spheroidal & 49.8 & 12.4 & 0.24 \\
\hline E. Hermmendoff 503 & 46.7 & 49.4 & 0.94 & oblate spheroidal & 49.0 & 11.3 & 0.22 \\
\hline C.M.B. Correia et al. 11 & 46.0 & 50.8 & 0.90 & oblate spheroidal & 48.6 & 11.8 & 0.24 \\
\hline \multicolumn{8}{|l|}{ V. phosphorica } \\
\hline J.P.P. Carauta 4833 et al. & 45.6 & 50.1 & 0.91 & oblate spheroidal & 51.2 & 15.0 & 0.29 \\
\hline J.P.P. Carauta 4781 et al. & 46.9 & 50.6 & 0.92 & oblate spheroidal & 51.9 & 15.0 & 0.28 \\
\hline E.F. Paciornil 55 & 46.9 & 52.1 & 0.90 & oblate spheroidal & 52.7 & 16.4 & 0.31 \\
\hline W. Hoehne 1417 & 47.3 & 50.9 & 0.92 & oblate spheroidal & 51.5 & 14.9 & 0.28 \\
\hline \multicolumn{8}{|l|}{ V. puberula } \\
\hline A. Custodio-Filho 285 & 49.4 & 54.0 & 0.91 & oblate spheroidal & 51.5 & 13.0 & 0.25 \\
\hline \multicolumn{8}{|l|}{ V. rigiophylla } \\
\hline A. Silveira 1257 & 46.9 & 51.4 & 0.91 & oblate spheroidal & 51.0 & 14.5 & 0.28 \\
\hline \multicolumn{8}{|l|}{ V. viscidula } \\
\hline M. Barreto 4233 & 52.3 & 57.4 & 0.91 & oblate spheroidal & 51.4 & 20.1 & 0.39 \\
\hline R. Romero et al. 2292 & 49.8 & 55.5 & 0.89 & oblate spheroidal & 54.7 & 20.5 & 0.37 \\
\hline \multicolumn{8}{|l|}{ V. westiniana } \\
\hline A.F. Regnell 1245 & 41.7 & 46.0 & 0.90 & oblate spheroidal & 45.7 & 15.7 & 0.34 \\
\hline R. Romero et al. 3289 & 42.2 & 46.5 & 0.90 & oblate spheroidal & 49.0 & 15.7 & 0.32 \\
\hline V.C. Souza et al. 2173 & 43.5 & 47.8 & 0.91 & oblate spheroidal & 47.5 & 16.0 & 0.33 \\
\hline W. Hoehne 4081 & 42.6 & 47.0 & 0.90 & oblate spheroidal & 46.2 & 15.8 & 0.34 \\
\hline
\end{tabular}



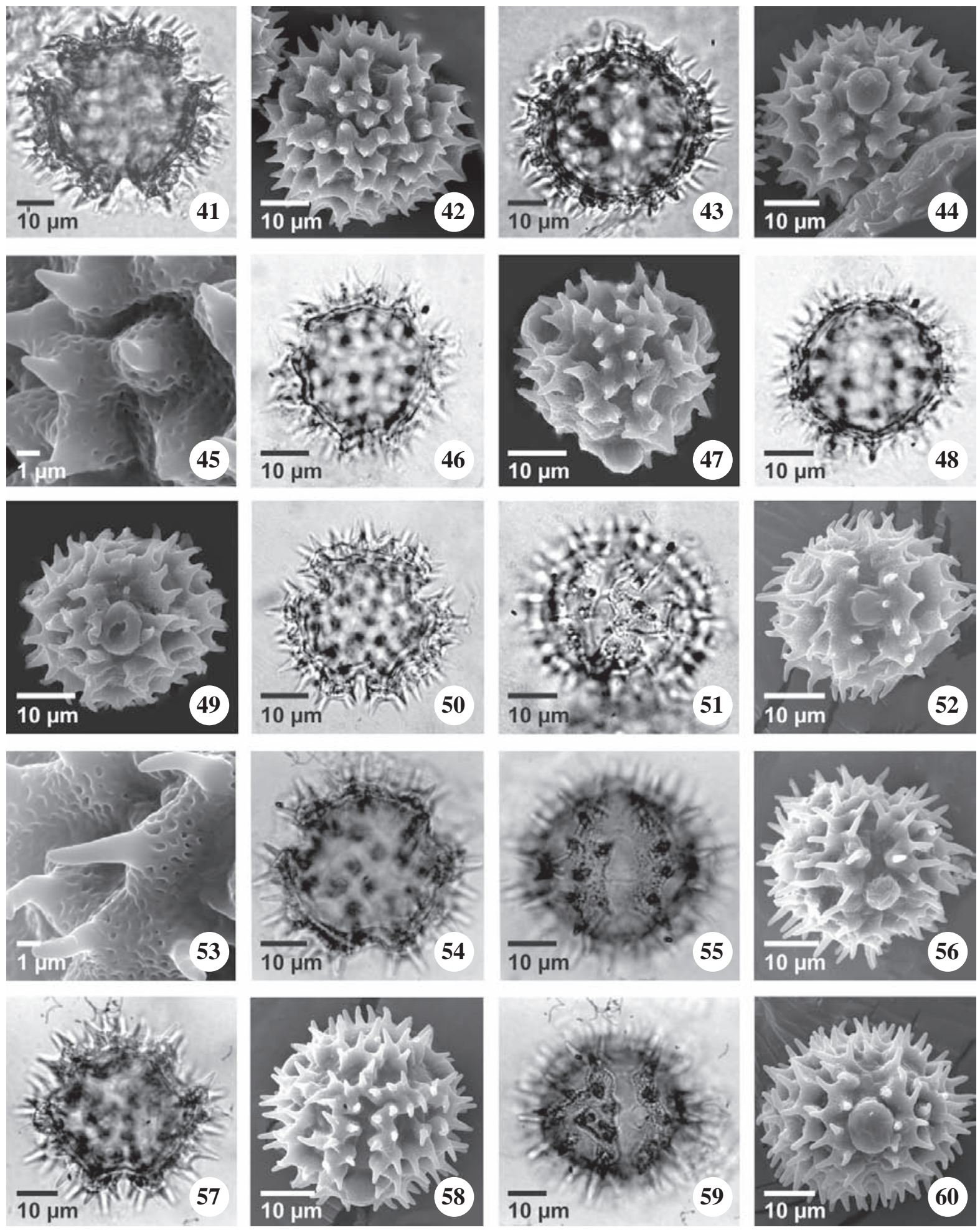

Figures 41-60. LM and SEM photomicrographs of the pollen grains of Vernonanthura. 41-45. V. lucida (G. Hatschbach et al. 67442) - Polar view. 41. Cross section. 42. Surface of apocolpium. Equatorial view. 43. Cross section. 44. Aperture. 45. Detail of surface. 46-49. V. mariana (R. Esteves \& V. Esteves 427). Polar view. 46. Cross section. 47. Surface of apocolpium. Equatorial view. 48. Cross section. 49. Aperture. 50-53. V. membranacea (G. Hatschbach et al. 72203) - Polar view. 50. Cross section. Equatorial view. 51-52. Aperture. 53. Detail of surface. 54-56. V. montevidensis (V.C. Souza \& C.M. Sakuragui 4328) - Polar view. 54. Cross section. Equatorial view. 55-56. Aperture. 57-60. V. mucronulata (J. Vidal s.n. R113249) - Polar view. 57. Cross section. 58. Surface of apocolpium. Equatorial view. 59-60. Aperture. 

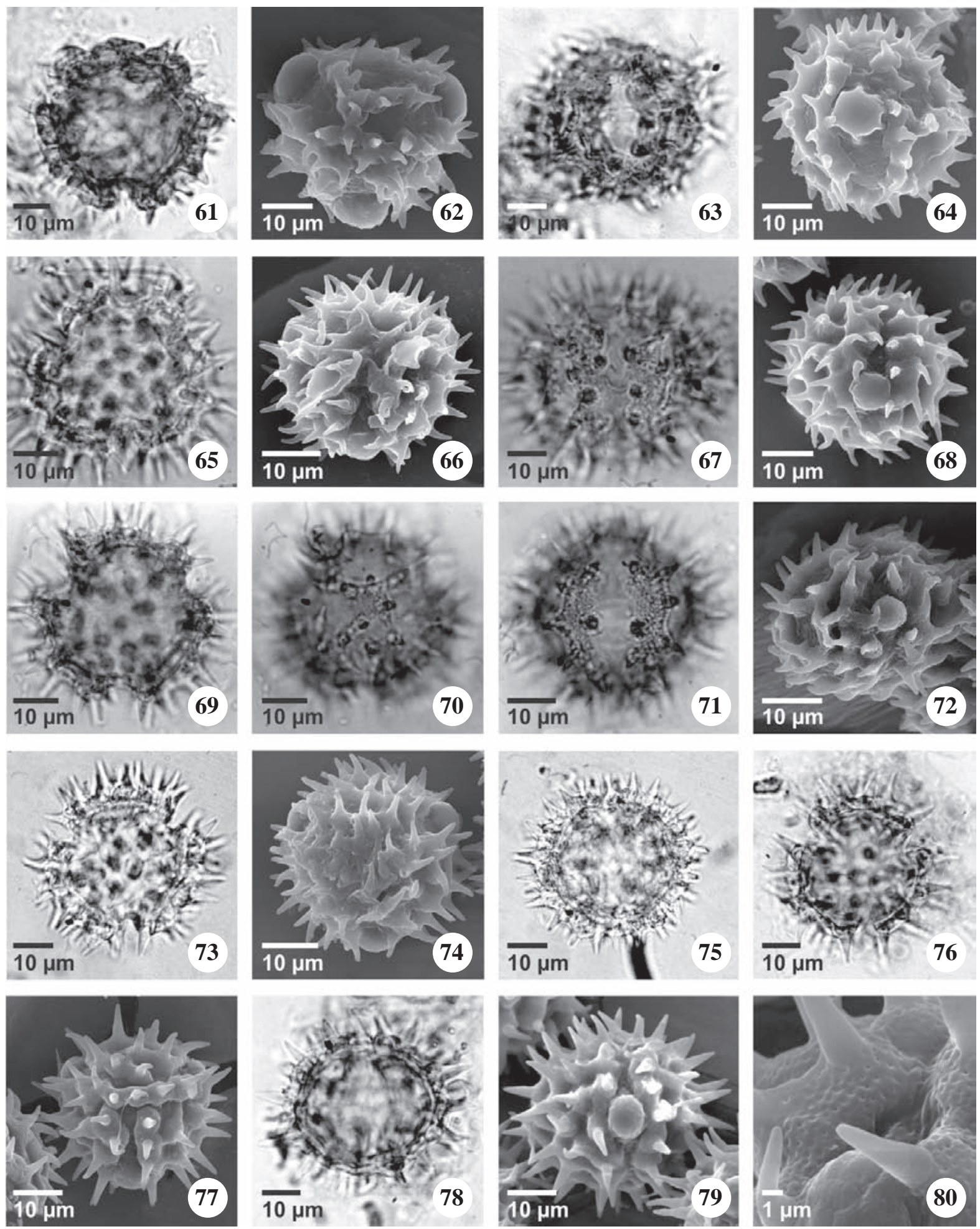

Figures 61-80. LM and SEM photomicrographs of the pollen grains of Vernonanthura. 61-64. V. oligolepis (C.A. de M. Scaramuzza \& V.C. Souza 2701) - Polar view. 61. Cross section. 62. Surface of mesocolpium. Equatorial view. 63-64. Aperture. 65-68. V. paludosa (P. Dusén 454) - Polar view. 65. Cross section. 66. Surface of apocolpium. Equatorial view. 67-68. Aperture. 69-72. V. petiolaris (J. Vidal 182) - Polar view. 69. Cross section. 70. Surface of apocolpium. Equatorial view. 71-72. Aperture. 73-75. V. phosphorica (G. Hatschbach et al. 65250) - Polar view. 73. Cross section. 74. Surface of apocolpium. Equatorial view. 75. Cross section. 76-80. V. puberula (Glaziou 17022) - Polar view. 76. Cross section. 77. Surface of apocolpium. Equatorial view. 78. Cross section. 79. Aperture. 80. Detail of surface. 

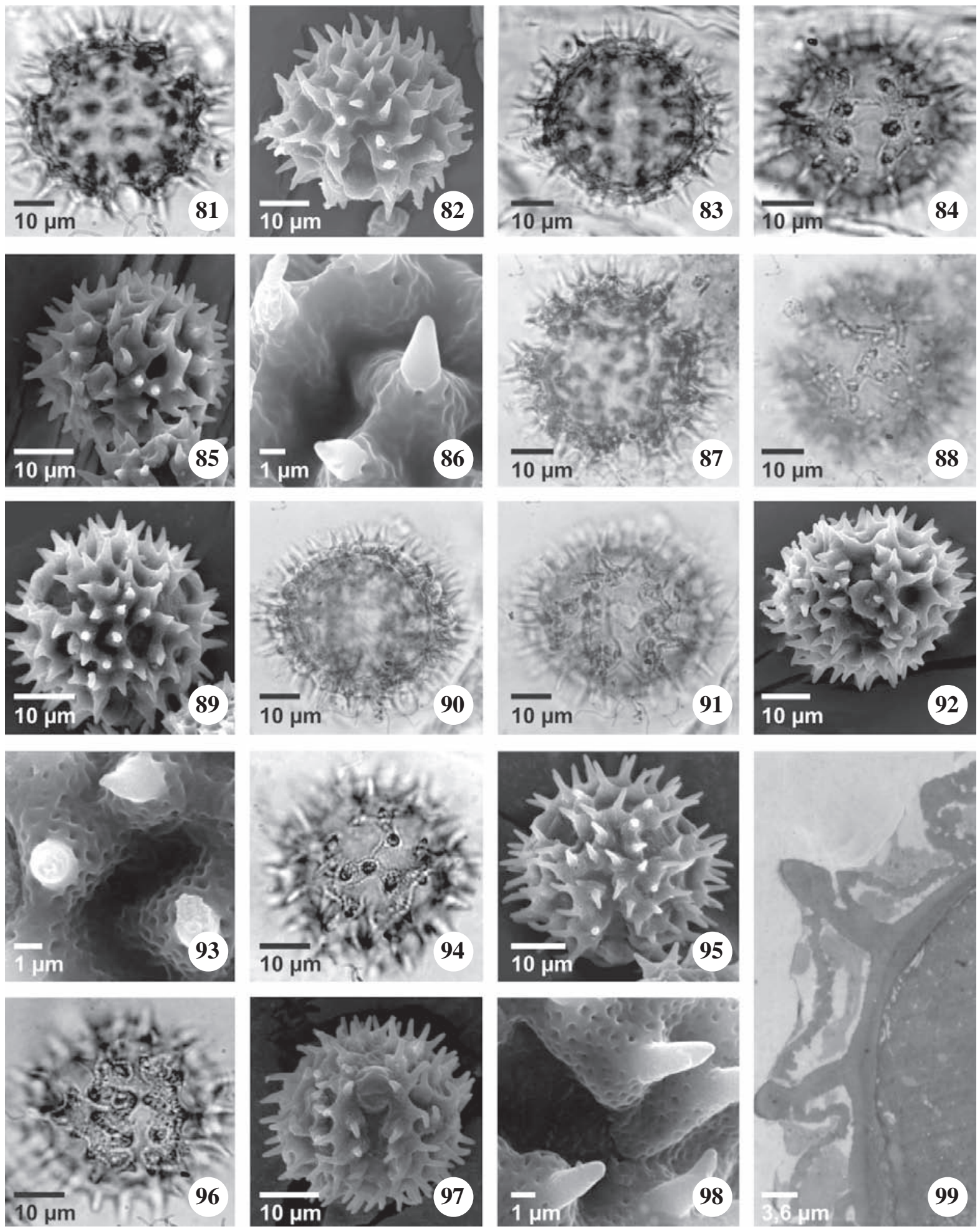

Figures 81-99. LM and SEM photomicrographs of the pollen grains of Vernonanthura. 81-86. V. rigiophylla (J.N. Nakajima et al. 1214) - Polar view. 81. Cross section. 82. Surface of mesocolpium. Equatorial view. 83. Cross section. 84-85. Aperture. 86. Detail of surface. 87-93. V. viscidula (Glaziou 15060) - Polar view. 87. Cross section. 88-89. Surface of apocolpium. Equatorial view. 90. Cross section. 91-92. Aperture. 93. Detail of surface. 94-99. V. westiniana (M. Frigoleto s.n. GUA47702) - Polar view. 94-95. Surface of apocolpium. Equatorial view. 96-97. Aperture. 98. Detail of surface. 99. Detail of exine estructure (TEM). 
and equatorial diameters within the limits of variation of its respective standard specimen. Regarding the shape, only the following specimens showed differences considering the standard material: V. chamaedrys
(Mello Barreto 1028, H.F. Leitão Filho et al. 19415), V. divaricata (G. Hatschbach et al. 59707), V. ignobilis (G. Hatschbach et al. 27862) and V. lindbergii (J. Augusto s.n. MBM188056).

Key to the species of Vernonanthura based on pollen morphology

1. Large sized pollen grains $(50.0-100.0 \mu \mathrm{m})$

2. Very small polar area $(<0.25 \mu \mathrm{m})$

3. Endoaperture with a median constriction; continuous muri tending towards the lophate pattern; walls distributed uniformly in the apocolpus V. crassa

3. Endoaperture without a median constriction; discontinuous wall not tending towards the lophate pattern; muri in the apocolpus only delimiting the apertures V. oligolepis

2. Small polar area $(0.25-0.50 \mu \mathrm{m})$

4. Pollen grains with IC $95 \% \geq 53.9 \mu \mathrm{m}$, colpus $>25.0 \mu \mathrm{m}$ long; muri very sinuous; continuous V. lucida

4. Pollen grains with IC $95 \%<53.6 \mu \mathrm{m}$; colpus $\leq 24.0 \mu \mathrm{m}$; muri slightly sinuous; discontinuous

5. Pollen grains with IC 95\% DP $=52.7-53.6 \mu \mathrm{m}$; endoapertures lightly lolongate $(11.8 \times 11.2 \mu \mathrm{m})$; without a median constriction; walls in the apocolpium only delimiting the aperture V. ignobilis

5. Pollen grains with IC 95\% DP $=50.1-51.9 \mu \mathrm{m}$; endoaperture clearly lalongate $(6.1 \times 10.6 \mu \mathrm{m})$; with a median constriction; muri distributed uniformly in the apocolpium V. viscidula

1. Medium sized pollen grains $(25.0-50.0 \mu \mathrm{m})$

6. Very small polar area $(\leq 0,25 \mu \mathrm{m})$

7. Endoapertures with a median constriction; colpus $c a$. $27.6 \mu \mathrm{m}$ long; interspinal distance ca. $10.6 \mu \mathrm{m}$ V. mucronulata

7. Endoaperture without a median constriction; colpus $<26,7 \mu \mathrm{m}$ long; interspinal distance $\leq 10,0 \mu \mathrm{m}$

8. Pollen grains with IC 95\% DP $=48.8-50.0 \mu \mathrm{m}$; muri tending towards the lophate pattern; spine $>8.0 \mu \mathrm{m}$ long V. puberula

8. Pollen grains with IC $95 \% \mathrm{DP} \leq 47.1 \mu \mathrm{m}$; muri not tending towards the lophate pattern; spine $\leq 6.4 \mu \mathrm{m}$ long

9. Pollen grains with IC 95\% DP $=41.4-42.2 \mu \mathrm{m}$; endoapertures $>14.0 \mu \mathrm{m}$ wide; spine $<5.5 \mu \mathrm{m}$ long V. ferruginea

9. Pollen grains with IC $95 \% \mathrm{DP} \geq 43.8 \mu \mathrm{m}$; endoapertures $\leq 9.0 \mu \mathrm{m}$ wide; spine $>6.0 \mu \mathrm{m}$ long V. paludosa, V. petiolaris

6. Small polar area $(>0.25 \mu \mathrm{m})$

10. Pollen grains with muri tending towards the lophate pattern

11. Muri slightly sinuous

12. $\mathrm{DP}=40.7-41.8 \mu \mathrm{m}$ V. divaricata, V. membranacea

12. DP $>45.0 \mu \mathrm{m}$ or $<39.7 \mu \mathrm{m}$

13. Endoapertures with a median constriction; spines $\leq 5.0 \mu \mathrm{m}$ long V. cuneifolia

13. Endoapertures without a median constriction; spines $\geq 7.5 \mu \mathrm{m}$ long V. mariana

11. Muri very sinuous

14. Muri continuous; $\mathrm{DP}=45.3-46.1 \mu \mathrm{m}$ V. montevidensis

14. Muri discontinuous; DP $>47.5 \mu \mathrm{m}$ or $<42.7 \mu \mathrm{m}$ 15. $\mathrm{DP}=47.5-48.5 \mu \mathrm{m}$; endoapertures with a median constriction V. rigiophylla 15. $\mathrm{DP}=41.8-42.7 \mu \mathrm{m}$; endoapertures without a median constriction V. westiniana 
10. Pollen grains with muri slightly or very sinuous and not tending towards the lophate pattern

16. Muri very sinuous, continuous

17. $\mathrm{DP}=43.5-44.2 \mu \mathrm{m}$ V. brasiliana

17. $\mathrm{DP}=48.2-49.4 \mu \mathrm{m}$ V. chamaedrys

16. Muri slightly sinuous discontinuous

18. DP = 37.3-38.2 $\mu \mathrm{m}$; colpus ca. $20.8 \mu \mathrm{m}$ long; spine $c a$. $3.8 \mu \mathrm{m}$ long V. discolor

18. $\mathrm{DP} \geq 44.5 \mu \mathrm{m}$; colpus $>24.3 \mu \mathrm{m}$ long; spine $>7.3 \mu \mathrm{m}$ long

19. $\mathrm{DP}=44.5-45.0 \mu \mathrm{m}$ V. lindbergii

19. $\mathrm{DP}=46.1-46.9 \mu \mathrm{m}$ V. phosphorica

\section{Discussion}

The 22 species of Vernonanthura presented homogeneous pollen grains regarding the shape (oblate spheroidal), the type of aperture (3 colpori) and the organization of the ornamentation patterns. They differ in size (medium or large), polar area (very small or small), endoapertures configurations (with or without a median constriction) and ornamentation details. Based on these differences, mostly species can be distinguished, with the exception of Vernonanthura paludosa $\times V$. petiolaris and $V$. divaricata $\times V$. membranacea .

There are few studies about the pollen morphology of Vernonia species moved to the genus Vernonanthura (Robinson 1992).

Stix (1960) recognized 42 different pollen types in the Asteraceae based on the ornamentation and structure of the exine. Within the Lychnophora type was included Vernonia diffusa Less. (= Vernonanthura divaricata) that has been analyzed in this work. The species examined here shows similar characteristics as the Lychnophora type designated by Stix (1960).

Kingham (1976) studied the pollinic morphology of the Vernonieae and established six pollen types based on the exine organization. Among the analyzed species, Vernonia brasiliana (L.) Druce (= Vernonanthura brasiliana) was placed in type VI by the author (subechinolophate to echinate, tricolporate with microperforated muri). The author further emphasized that there was a possibility of a subdivision for the type, based on the organization of the spines (these may be regularly or irregularly spaced) and by the number of perforations in the muri. The present study confirms the classification of Vernonanthura brasiliana in Kingham's (1976) type VI, and the possibilities of the subdivisions suggested by him. The pollinic key here elaborated confirms Kingham's observation (1976) regarding the differences in organization of the exine (muri tending or not towards the lophate pattern).
Keeley \& Jones (1977) studied the species of Vernonia from New World and described three pollen types called A, B and C. Some years later, Jones (1979) recognized these three types and described an additional type named "D” (pollen grains echinolophate, triporate, discontinuous tectum, microperforated). The author affirmed the occurrence of the types A and B in the subsection of Vernonia chamaedrys Less. and classified as type A the species $V$. chamaedrys, $V$. crassa, $V$. cuneifolia, V. montevidensis, V. mucronulata, V. puberula, $V$. rigiophylla and $V$. viscidula, all them analyzed in this study. For the subsection Nudiflorae, the author reports only the type A, in which, among others, lie the species $V$. brasiliana, V. mariana and V. westiniana. The present study confirms the classification of the pollen grains as suggested by Jones (1979) based on the ornamentation pattern and the apertural type, according to which the Vernonanthura species present type A pollen grains.

Robinson (1999) pointed out that pollen morphology is a useful characteristic for the taxonomy of the Vernonieae and, according to the author, it is inexplicable that taxons are still described without pollen details. However, Robinson (1999) applied imprecise terms in his brief descriptions, such as rhizomate form (= ramification of the columellae) and baculate of the crests (= muri) of the lophate exine. The author suggested that the pollen type A, found in Vernonanthura, is apparently, a reversion of the lophate shape, which was maintained on some groups of Neotropical Vernonieae, such as Vernonia/Vernonanthura. In our study, the proposition for Vernonanthura made by Robinson (1999) was not supported, because some species presented typical subechinolophate pollen grains, and, in others, a tendency towards the lophate pattern. Even so, at the moment, it can not be confirmed which of the two types is more basal, as phylogenetic studies will possibly be required for elucidation.

Mendonça \& Gonçalves-Esteves (2000) described the pollen morphology of some species of the tribe 
Vernonieae occurring in the Carapebus "restinga” in Rio de Janeiro. Among studied species, Vernonia beyrichii Less. (Vernonanthura beyrichii (Less.) H. Rob.) is outstanding as it presents large pollen grains, isopolar, prolate spheroidal, triangular amb, small polar area, tricolporate, subechinolophate. This species seems very similar to Vernonanthura ignobilis, due to the pollen size and the small polar area, but it can be separated by the prolate spheroidal shape, endoapertures notably lalongate $(10 \times 7.0 \mu \mathrm{m})$ and the slightly sinuous muri tending towards a lophate pattern, distributed uniformly in the apocolpium.

DeVore et al. (2000), using TEM, accepted the definitions made by Skvarla et al. (1977) about the "anthemoide" pattern (non-caveate exine, long basal columella, above which it has shorter columellae alternating with internal tectum). According to authors, Lactuceae, Liabeae and Vernonieae present the "liabioideantemoide" pattern as derived from an ancestral caveate shared with Asteroideae. The "antemoide" pattern evolved inside the subfamily Asteroideae. In our study, Vernonanthura westiniana, the single species analyzed with TEM, showed a pattern similar to the "antemoide", mainly due to the lack of cava.

In an analysis of the pollen variability of the plants from Campos de Jordão, Melhem et al. (2003) described 41 species of Compositae, including Vernonia polyanthes Less. (Vernonanthura phosphorica) and Vernonia westiniana Less. (Vernonanthura westiniana). Confronting the results found by the authors against those observed here, differences were ascertained, mainly in relation to the ornamentation. The authors described the exine as lophate, while in this study the sexine was considered subechinolophate. The authors suggested that the pollen of Vernonanthura phosphorica is large sized, and that the pollen of $V$. westiniana presents spherical shape. In our study, these species are considered medium size with an oblate spheroidal shape. However, the disparity in the results may be attributed to the incorrect taxonomic identification of the analyzed taxa.

Skvarla et al. (2005) described the pollen grains of eight North American species of Vernonia s.s. and three of Elephantopus. The authors concluded that the first genus presents subechinolophate exine with two types of columellae; in the first, the columellae are individual, solid, larger, ca. $2 \mu \mathrm{m}$ in diameter and support the spine; in the second type, the columellae are smaller, ca. $1 \mu \mathrm{m}$ diameter, less solid and do not support the spine. The pollen grains of the Vernonanthura species analyzed here, in both SEM and TEM (Vernonanthura westiniana), were similar (subechinolophate, tricolporate) to the
Vernonia s.s. described by Skvarla et al. (2005). Based on the results found, it is considered that the evaluation of the pollen grain, does not reinforce the separation of those species currently maintained by Robinson (1988, 1994) in Vernonia s.s. because Vernonanthura presents subechinolophate and tricolporate pollen.

From the obtained results, Vernonanthura was considered an euripolinic genus whose pollen grains presented typical morphological characteristics, mainly regarding to the ornamentation, the size of the pollen grains and the features of the aperture, and all them may serve as further tools in delimitation of the species.

Acknowledgements - The authors thank MSc. Diego Knop for the english review; to Laboratório de Ultraestrutura Celular, of the Institute of Biophysics, Universidade Federal do Rio de Janeiro (UFRJ), with special thank to Noêmia Rodrigues Gonçalves, technician in scanning electron microscopy. Furthermore, we wish to thank Faperj (proc. E-26/171.071/03); CNPq (proc. 481595/2004-0) and the third author's productivity scholarship; and Capes for the first author's doctorate scholarship.

\section{References}

DEVORE, M.L., ZHAO, Z., JANSEN, R.K. \& SKVARLA, J. 2000. Utility of trends in pollen morphology for phylogenetic analyses: an exemple using subfamilies Barnadesioidese and Cichoroideae (Asteraceae). In Pollen and Spores: morphology and biology (M.M. Harley, C.M. Morton and S. Blackmore, eds.). Royal Botanic Gardens, Kew, p.399-412.

ERDTMAN, G. 1952. Pollen morphology and plant taxonomy - Angiosperms. Almqvisit \& Wiksel, Stockholm.

FAEGRI, G. \& IVERSEN, J. 1966. Textbook of modern pollen analysis. $2^{\text {nd }}$ ed. Scandinavian University Books, Copenhagen.

HOLMGREN, P.K., HOLMGREN, N.H. \& BAINETT, L.G. 1990. Index Herbariorum. Part 1: The Herbaria of the world. $8^{\text {nd }}$ ed., New York Botanical Garden, New York.

JONES, S.B. 1977. Vernonieae - systematic review. In The biology and chemistry of the Compositae (V.H. Heywood, J.B. Harbone \& B.L. Turner, eds.). Academic Press., London, p.503-521.

JONES, S.B. 1979. Synopsis and pollen morphology of Vernonia (Compositae: Vernonieae) in the New World. Rhodora 81:425-447.

KEELEY, S.C. \& JONES, S. 1977. Taxonomic implications of external pollen morphology to Vernonia (Compositae) in the West Indies. American Journal of Botany 64: 576-584.

KINGHAM, D.L. 1976. A study of the pollen morphology of Tropical African and certain other Vernonieae (Compositae). Kew Bulletin 31:9-26. 
MELHEM, T.S., CRUZ-BARROS, M.A.V., CORRÊA, A.M.S., MAKINO-WATANABE, H., SILVESTRECAPELATO, M.S.F. \& GONÇALVES-ESTEVES, V. 2003. Variabilidade polínica em plantas de Campos de Jordão (São Paulo, Brasil). Boletim do Instituto de Botânica 16:9-104.

MENDONÇA, C.B.F. \& GONÇALVES-ESTEVES, V. 2000. Morfologia polínica de algumas espécies da tribo Vernonieae (Compositae Giseke) ocorrentes na restinga de Carapebus, Rio de Janeiro. Hoehnea 27:131-142.

PUNT, W., BLACKMORE, S., NILSSON, S. \& LE THOMAS, A. 2007. Glossary of pollen and spore terminology. Review of Paleobotany and Palynology 143:1-81.

ROBINSON, H. 1988. Studies in the Lepidaploa complex (Vernonieae: Asteraceae) V - The new genus Chrysolaena. Proceedings of the Biological Society of Washington 101:952-958.

ROBINSON, H. 1992. A new genus Vernonanthura H. Rob. (Vernonieae: Asteraceae). Phytologia 73:65-76.

ROBINSON, H. 1994. New combinations in American Vernonieae (Asteraceae). Phytologia 76:27-29.
ROBINSON, H. 1999. Generic and subtribal classification of American Vernonieae. Smithsonian Contributions to Botany, Washington.

SALGADO-LABOURIAU, M.L. 1973. Contribuição à palinologia dos cerrados. Academia Brasileira de Ciências, Rio de Janeiro.

SKVARLA, J.J., TURNER, B.L., PATEL, V.C. \& TOMB, A.S. 1977. The pollen morphology in the Compositae and in morphologically related families. In The biology and chemistry of the Compositae (V.H. Heywood, J.B. Harborne \& B.L.Turner, eds). Academic Press., London, p.141-248.

SKVARLA, J.J., DEVORE, M.L. \& CHISSOE, W.F. 2005. Lophate sculpturing of Vernonieae (Compositae) pollen. Review Palaeobotany and Palynology 133: 51-68.

STIX, E. 1960. Pollenmorpholosgische untersuchungen na Compositen. Grana Palynologica 2:41-114.

STUTTS, J.G. 1988. Taxonomic revision of Vernonia subsect. Chamaedrys (Compositae: Vernonieae). Rhodora 90: 37-99. 